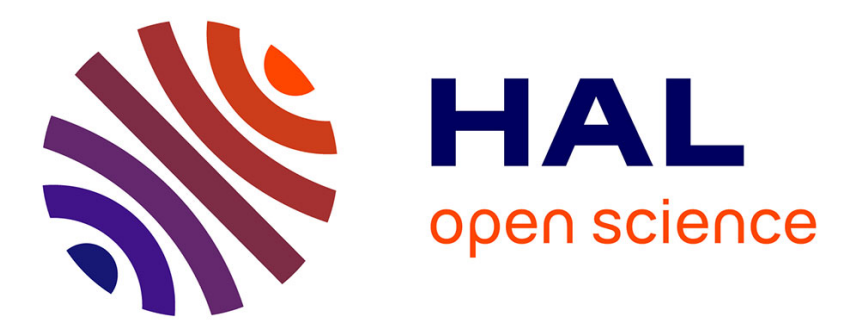

\title{
On-line estimation of a stability metric including grip conditions and slope: Application to rollover prevention for all-terrain vehicles
}

\author{
M. Richier, R. Lenain, Benoît Thuilot, C. Debain
}

\section{- To cite this version:}

M. Richier, R. Lenain, Benoît Thuilot, C. Debain. On-line estimation of a stability metric including grip conditions and slope: Application to rollover prevention for all-terrain vehicles. IROS'11, IEEE/RSJ International conference on intelligent robots and systems, Sep 2011, San Francisco, United States. p. - p. hal-00665035

\section{HAL Id: hal-00665035 https://hal.science/hal-00665035}

Submitted on 1 Feb 2012

HAL is a multi-disciplinary open access archive for the deposit and dissemination of scientific research documents, whether they are published or not. The documents may come from teaching and research institutions in France or abroad, or from public or private research centers.
L'archive ouverte pluridisciplinaire HAL, est destinée au dépôt et à la diffusion de documents scientifiques de niveau recherche, publiés ou non, émanant des établissements d'enseignement et de recherche français ou étrangers, des laboratoires publics ou privés. 


\title{
On-line estimation of a stability metric including grip conditions and slope: Application to rollover prevention for All-Terrain Vehicles
}

\author{
Mathieu Richier ${ }^{1}$, Roland Lenain ${ }^{1}$, Benoit Thuilot $^{2,3}$, Christophe Debain ${ }^{1}$ \\ ${ }^{1}$ Cemagref, 24 avenue des Landais, 63172 Aubière, France \\ ${ }^{2}$ Clermont Université, Université Blaise Pascal, LASMEA, BP 10448, 63000 Clermont-Ferrand, France \\ 3 CNRS, UMR 6602, LASMEA, 63177 Aubière, France \\ \{mathieu.richier, roland.lenain, christophe.debain\}@cemagref.fr＿ benoit.thuilot@lasmea.univ-bpclermont.fr
}

\begin{abstract}
Rollover is the principal cause of serious accidents for All-Terrain Vehicles (ATV), especially for light vehicles (e.g. quad bikes). In order to reduce this risk, the development of active devices, contributes a promising solution. With this aim, this paper proposes an algorithm allowing to predict the rollover risk, by means of an on-line estimation of a stability criterion. Among several rollover indicators, the Lateral Load Transfer (LLT) has been chosen because its estimation needs only low cost sensing equipment compared to the price of a light ATV. An adapted backstepping observer associated to a bicycle model is first developed, allowing the estimation of the grip conditions. In addition, the lateral slope is estimated thanks to a classical Kalman filter relying on measured acceleration and roll rate. Then, an expression of the LLT is derived from a roll model taking into account the grip conditions and the slope. Finally, the LLT value is anticipated by means of a prediction algorithm. The capabilities of this system are investigated thanks to full scale experiments with a quad bike.
\end{abstract}

\section{INTRODUCTION}

Quad bikes are more and more popular, for both leisure activities and work. For example in the agricultural context, quad bikes are frequently used because of their conception and the consequential off-road capabilities. Nevertheless their geometric characteristics increase the risk of unsafe or unstable situations. In addition, most ATV users do not have necessarily the required qualification to drive their vehicle under off-road conditions. Consequently, the number of accidents is rising. Among serious accidents, the rollover situation is preponderant as it has been confirmed in many studies (almost $50 \%$ of ATV crashes as mentioned in [1] and [2]). Therefore the design of security systems to ensure the stability of ATV is a relevant topic.

Such devices have already been developed for on-road vehicles, such as the Electronic Stability Program (ESP) [3] or steering and braking control [4] which improve the control of the trajectory. In the same way, in order to improve the vehicle stability, algorithms which estimate the sliding parameters (slip angle, tire cornering stiffnesses) and roll angle (preponderant variables for the risk of rollover) have been designed ([5], [6] and [7]). These algorithms use linear tire-ground models, which are well adapted since grip conditions do not exhibit large variations. Nevertheless those devices are not transposable to rough terrain applications due to the highly variable environment conditions.
For the off-road context, some stability systems have been designed, mainly dedicated to mobile robots. The principal aim is to ensure their integrity. A first case is to consider only geometric stability conditions ([8] and [9]). This is suitable in a low speed context, as dynamic effects are neglected. At high speed, other revelant researches have been achieved like [10] and [11]. The main limitation to transpose above systems to an ATV in order to prevent the risk of rollover, is the expensive necessary sensing equipment, compared to the vehicle price. Therefore, highly accurate INS, RTK GPS etc, employed by most of the previously cited systems, are not adapted in this application. Moreover, the accessibility of GPS data can not be ensured when the ATV moves in natural environment (trees, mountains, building etc).

In previous work [12], a rollover risk prevention system dedicated to high speed ATVs has been proposed, based on a low cost sensing equipment. It estimates on-line the tire-ground friction and is thus adaptive to changing grip conditions. Among several rollover indicators [13], the Lateral Load Transfer (LLT) has been chosen as a revelant stability criterion. It will be used in this paper as well. Although the algorithm efficiency has already been proved, two main limitations have been left. First, the grip conditions can not be updated in straight line due to a singularity in the algorithm. Secondly, a flat ground has been assumed, which constitutes an important limitation with respect to off-road applications. Consequently, in order to improve the reliability of this approach, we propose an extension in modeling and in grip condition estimation. Using a 3axes accelerometer/gyrometer, a Doppler radar and a steering angle sensor, the LLT can be predicted whatever the grip conditions and the slope are. More precisely, a bicycle model is associated to an adapted backstepping observer, allowing to access the sliding parameters, while the slope is estimated using a classical Kalman filter relying on the lateral-vertical accelerations and the roll rate. These estimations are then fed into a prediction algorithm using a roll model, allowing the LLT anticipation. The efficiency of this system has been tested through full scale experiments.

The paper is organized as follows: first, the vehicle modeling (yaw and roll projection) associated with the tire-ground contact model are depicted, allowing the rollover metric computation. As the LLT expression (derived from the roll model) requires the knowledge of the sliding parameters, 
a new adapted backstepping observer is developed in the second part. In the same part, a prediction algorithm allowing LLT value anticipation is described. Finally, full scale experiments (with a commercial quad bike) are presented to discuss the applicability of the proposed approach.

\section{ROLLOVER METRIC COMPUTATION}

\section{A. Dynamic model}

In order to achieve on-line LLT computation when the ground is uneven, the global vehicle modeling depicted on Fig. 1 is considered. The dynamical model of vehicle is split into two models. The first model represents a yaw 2D projection (shown on Fig.1(a)) assuming a flat ground. Nevertheless, a lateral force $\left(P_{1}\right)$ is added to take into account the effect of slope on yaw dynamics. Relying on the state observer described in section III, this model enables the estimation of the sliding parameters (sideslip angles $\beta, \alpha_{f}$, $\alpha_{r}$ and lateral forces $F_{f}$ and $F_{r}$ ), which influence the risk of rollover. These sliding conditions are then injected into the second model: a roll 2D projection (shown on Fig.1(b)) used to estimate the LLT.

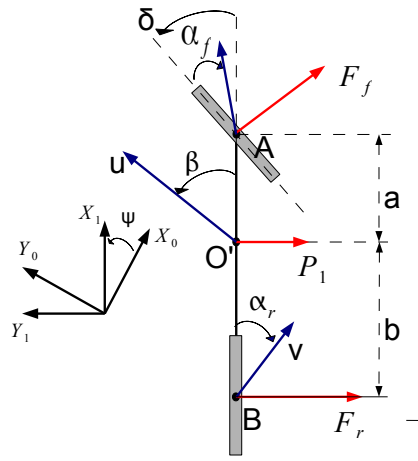

(a) Yaw projection

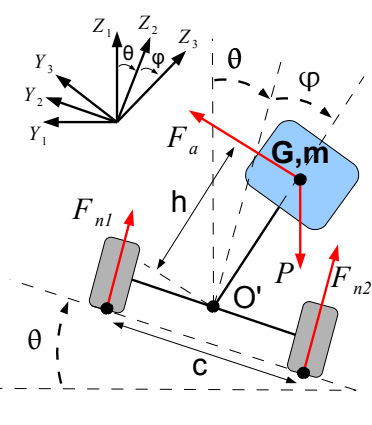

(b) Roll projection
Fig. 1. Dynamic bicycle model with sliding parameters.

The variables used in the sequel, reported on Fig.1(a) and Fig.1(b), are listed below:

- $\psi$ is the vehicle yaw angle,

- $\theta$ is the bank angle of the terrain in the roll projection,

- $\delta$ is the steering angle,

- $v$ is the linear velocity at the center of the rear axle,

- $u$ is the linear velocity at the roll center $O^{\prime}$,

- $a$ and $b$ are the front and rear vehicle half-wheelbases,

- $c$ is the vehicle track,

- $h$ is the distance between the roll center and the vehicle center of gravity $G$,

- $I_{x}, I_{y}, I_{z}$ are the roll, pitch and yaw moments of inertia,

- $P=m g$ is the gravity force on the suspended mass $m$, with $g$ denoting the gravity acceleration,

- $P_{1}=m g \sin (\theta)$ is the influence of the gravity force on the lateral dynamics,

- $F_{n 1}$ and $F_{n 2}$ are the normal component of the tireground contact forces on the vehicle left and right sides,
- $F_{a}(\varphi)$ is a restoring-force parametrized by $k_{r}$ and $b_{r}$, the roll stiffness and damping coefficients:

$$
\overrightarrow{F_{a}}=\frac{1}{h}\left(k_{r} \varphi+b_{r} \dot{\varphi}\right) \overrightarrow{y_{2}}
$$

where $\varphi$ is the roll angle of the suspended mass associated to the roll dynamics, depicted on Fig.1. In section II-D, a way to calculate $\varphi$ is given.

\section{B. Contact model}

The forces $F_{r}$ and $F_{f}$ acting on lateral dynamics widely depend on grip conditions. As a result, a tire-ground model is mandatory. Among several models describing the sliding phenomena (such as Pacejka or LuGre model [14], [15]), the linear model (2) is considered:

$$
\begin{aligned}
& F_{f}=C_{f}(.) \alpha_{f} \\
& F_{r}=C_{r}(.) \alpha_{r}
\end{aligned}
$$

Its main advantage lies in the few numbers of parameters to be known. Nevertheless in order to take into account the nonlinearity of the contact and the variations of grip conditions, cornering stiffnesses $\left(C_{f}(\right.$.$\left.) and C_{r}().\right)$ are considered as varying. They are on-line adapted thanks to the observer detailed in section III.

\section{Motion equations in yaw frame}

Based on both the linear tire model and the bicycle model representation depicted on Fig.1(a), the equations of motion can be derived using the fundamental principle of the dynamic. In the yaw frame, longitudinal forces as well as roll and pitch motions are neglected. Moreover, sideslip angles are assumed to be small, and the influence of the bank angle is accounted via the addition of a gravity force in acceleration equations. Motion equations are then finally given by:

$$
\begin{aligned}
\ddot{\psi} & =\frac{-a C_{f} \alpha_{f}+b C_{r} \alpha_{r}}{I_{z}} \\
\dot{\beta} & =-\frac{C_{f} \alpha_{f}+C_{r} \alpha_{r}+m g \sin (\theta)}{u m}-\dot{\psi} \\
\alpha_{r} & =\beta-\frac{b \dot{\psi}}{u} \\
\alpha_{f} & =\beta+\frac{a \dot{\psi}}{u}-\delta \\
u & \approx v
\end{aligned}
$$

As this paper deals with dynamic LLT estimation, the velocity is assumed to be always strictly positive. As a result the condition $u \neq 0$ is always met.

\section{Roll motion and LLT computation}

The Lateral Load Transfer (LLT) represents the unbalanced repartition of the normal components of the tireground contact forces. It is mathematically defined as:

$$
L L T=\frac{F_{n 1}-F_{n 2}}{F_{n 1}+F_{n 2}}
$$

According to definition (4), the LLT reaches \pm 1 when two wheels on a vehicle's side lift off, which is representative of 
a rollover risk. In practice a threshold can be chosen above which the vehicle is considered in a hazardous situation. This threshold is chosen as $80 \%$ (classical value used in the literature) in order to define a safety margin.

Thanks to the fundamental principle of the dynamic applied to the roll model depicted Fig.1(b), and still assuming that the angles are small and moreover that $\ddot{\theta}<<\ddot{\varphi}$, dynamics equation for the roll angle $\varphi$ and for the normal forces are respectively given by (5), (6) and (7):

$$
\begin{aligned}
& \ddot{\varphi}=\frac{1}{h}\left[h \dot{\gamma}^{2} \varphi+h \dot{\psi}^{2} \gamma+u \dot{\psi}+\dot{u} \beta\right. \\
& \left.+u \dot{\beta}-\frac{k_{r} \varphi+b_{r} \dot{\varphi}}{m h}+g \sin (\theta)\right] \\
& F_{n 1}+F_{n 2}=m\left[-h \ddot{\varphi} \varphi-h \dot{\gamma}^{2}+g-u \dot{\psi} \theta\right. \\
& \left.-\frac{k_{r} \varphi+b_{r} \dot{\varphi}}{m h} \varphi-\dot{\psi}^{2} \gamma \theta+u \dot{\theta} \beta\right] \\
& F_{n 1}-F_{n 2}=\frac{2}{c}\left[I_{x} \ddot{\varphi}+\left(I_{z}-I_{y}\right) \dot{\psi}^{2} \frac{\sin (2 \gamma)}{2}\right. \\
& \left.-h \sin (\varphi)\left(F_{n 1}+F_{n 2}\right)\right]
\end{aligned}
$$

where $\gamma=\theta+\varphi$.

The LLT can then be inferred by reporting (6) and (7) into definition (4). Consequently, as soon as the roll angle $\varphi$ can be calculated using (5), the LLT can be evaluated thanks to the normal force expressions (6), and (7).

In view of (5), the calculation of $\varphi$ requires the knowledge of sideslip angle $(\beta)$ whose value depends on cornering stiffnesses $C_{f}$ and $C_{r}$ in view of (3). As quad bikes are expected to move on a natural and slippery ground, grip conditions have an important influence and are moreover varying. Since these variables can not be measured, their on-line adaptation is then required in order to obtain relevant estimation and prediction of the LLT. Therefore a backstepping observer has been designed to supply on-line their values. Moreover a prediction algorithm is mandatory, if the LLT has to be anticipate in order to prevent the hazardous situations.

\section{ROLLOVER PREVENTION}

\section{A. System overview}

The developed system aiming at ATV rollover prevention is summarized on Fig.2.

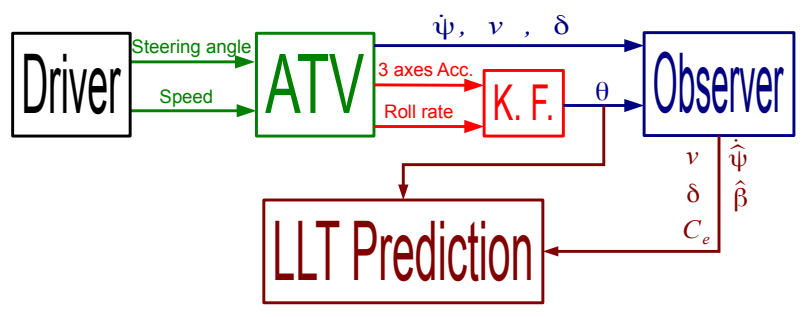

Fig. 2. Algorithm overview.
1) ATV box: The ATV is manually controlled, i.e. the driver specifies the vehicle speed $v$ and steering angle $\delta$. As described in the introduction, the measured data are the roll/yaw rate, the accelerations, the speed and the steering angle. The bank angle $\theta$ is indirectly estimated via a classical Kalman filter relying on the roll rate and lateral/vertical accelerations.

2) Observer box: Contact conditions are then on-line estimated, however for observability reasons, the two cornering stiffnesses can not be estimated separately, and are therefore considered to be equal to a global virtual cornering stiffness $C_{e}$. An estimation of the sideslip angle $\widehat{\beta}$ and yaw rate $\dot{\widehat{\psi}}$ is also supplied.

3) LLT prediction box: Relying on the measured and observed variables $\left(v, \delta, C_{e}, \dot{\hat{\psi}}\right.$ and $\left.\widehat{\beta}\right)$, future LLT values can be predicted on-line, in order to prevent the risk of rollover.

The observer and LLT prediction algorithms are more precisely described in the following sections.

\section{B. Observer design}

1) Observability : Using $C_{f}=C_{r}=C_{e}$, the motion equations (3) can be turned into the state space linear system (8).

$$
\begin{aligned}
\dot{X} & =\left[\begin{array}{ll}
a_{11} & a_{12} \\
a_{21} & a_{22}
\end{array}\right] \times X+\left[\begin{array}{cc}
b_{1} & 0 \\
b_{2} & b_{3}
\end{array}\right] \times U \\
Y & =C X
\end{aligned}
$$

where $X=[\dot{\psi}, \beta]^{T}, U=[\delta, \sin (\theta)]^{T}, C=[1,0]$ and with: $a_{11}=\frac{-\left(a^{2}+b^{2}\right) C_{e}}{v I_{z}}, a_{12}=\frac{(b-a) C_{e}}{I_{z}}, a_{21}=\frac{(b-a) C_{e}}{m v^{2}}-1$, $a_{22}=\frac{-2 C_{e}}{m v}, b_{1}=\frac{a C_{e}}{I_{z}}, b_{2}=\frac{C_{e}}{m v}, b_{3}=-\frac{g}{v}$

Kalman observability matrix $O_{o b s}$ for system (8) is:

$$
O_{o b s}=\left[\begin{array}{c}
C \\
C A
\end{array}\right]=\left[\begin{array}{cc}
1 & 0 \\
-\frac{\left(a^{2}+b^{2}\right) C_{e}}{v I_{z}} & \frac{(b-a) C_{e}}{I_{z}}
\end{array}\right]
$$

It can be checked that $O_{o b s}$ is a full rank matrix as soon as $a \neq b$ (which is always met on actual quad bikes) and $v \neq 0$. Under these assumptions, system (8) is observable and $\dot{\psi}$ as well as $\beta$ can be on-line estimated.

2) Backstepping approach: The observer equations associated with system (8) can be written as:

$$
\begin{aligned}
& \ddot{\widehat{\psi}}=a_{11} \dot{\widehat{\psi}}+a_{12} \widehat{\beta}+b_{1} \delta \\
& \dot{\widehat{\beta}}=a_{21} \dot{\hat{\psi}}+a_{22} \widehat{\beta}+b_{2} \delta+b_{3} \sin (\theta)
\end{aligned}
$$

where $\dot{\widehat{\psi}}$ and $\widehat{\beta}$ are respectively the observed yaw rate and sideslip angle. In order to compute the LLT, $\beta$ and $C_{e}$ have to be estimated from (10). With this aim, a backstepping approach composed of 3 steps is proposed. An overview is depicted on Fig.3.

a) First step: "Sideslip angle estimation"

The first step consists in treating $\widehat{\beta}$ as a control input (denoted $\bar{\beta})$, to be designed in order to impose the following dynamic on the observed yaw rate error $\tilde{\psi}$ :

$$
\ddot{\widetilde{\psi}}=\ddot{\psi}-\ddot{\widehat{\psi}}=K \dot{\widetilde{\psi}}, \quad K<0
$$




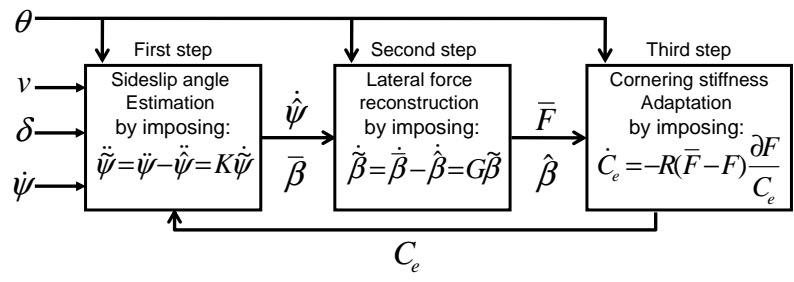

Fig. 3. Observer overview.

where $\ddot{\psi}$ is derived from the measured yaw rate. Injecting (11) into the first equation in (10) leads to the following expression for control variable $\bar{\beta}$ :

$$
\bar{\beta}=\frac{\ddot{\psi}-K \dot{\widetilde{\psi}}-a_{11}\left(C_{e}\right) \dot{\hat{\psi}}-b_{1}\left(C_{e}\right) \delta}{a_{12}\left(C_{e}\right)}
$$

Since $\bar{\beta}$ ensures that $\dot{\hat{\psi}}$ converges to the actual value $\dot{\psi}$ supplied by the gyrometer, $\bar{\beta}$ can be considered as a relevant estimation of the actual global sideslip angle.

b) Second step: "Lateral force reconstruction"

Referring to initial equations (3), the second equation in (10) can be rewritten in order to introduce a global lateral force $F$ defined as:

$$
F=C_{e}\left(\alpha_{f}+\alpha_{r}\right)
$$

It follows that:

$$
\begin{aligned}
\dot{\widehat{\beta}} & =-\frac{C_{e}\left(\alpha_{f}+\alpha_{r}\right)+m g \sin (\theta)}{v m}-\dot{\widehat{\psi}} \\
& =-\frac{F+m g \sin (\theta)}{v m}-\dot{\widehat{\psi}}
\end{aligned}
$$

Just as in the first step, $F$ is treated as a control input (denoted $\bar{F}$ ) to be designed to impose that $\widetilde{\beta}=\bar{\beta}-\widehat{\beta}$ converges to 0 with the following dynamic:

$$
\dot{\widetilde{\beta}}=\dot{\bar{\beta}}-\dot{\widehat{\beta}}=G \widetilde{\beta}, \quad G<0
$$

where $\dot{\bar{\beta}}$ is derived from $\bar{\beta}$. Injecting (15) into equation (14) leads to the following expression for control variable $\bar{F}$ :

$$
\bar{F}=-m v\left(\dot{\hat{\psi}}+\frac{g}{v} \sin (\theta)-G \widetilde{\beta}+\dot{\bar{\beta}}\right)
$$

Since $\bar{F}$ ensures that $\widehat{\beta}$ converges to the actual value $\bar{\beta}, \bar{F}$ can be considered as an estimation of the actual lateral force.

c) Third step: "Cornering stiffness adaptation"

The last step consists in adapting $C_{e}$ in order to ensure the convergence of $F$ to $\bar{F}$ as defined by equation (13). In view of (13), the adaptation of $C_{e}$ can not be achieved when $\left(\alpha_{f}+\alpha_{r}=0\right)$, which occurs especially when moving straight ahead. In order to avoid an adaptation interruption in such a case, a MIT rule adaptation [16] is proposed to obtain the convergence:

$$
\begin{aligned}
\dot{C}_{e} & =-R(\bar{F}-F) \frac{\partial F}{\partial C_{e}} \\
& =-R(\bar{F}-F)\left(\alpha_{f}+\alpha_{r}\right)
\end{aligned}
$$

with $R$ a strictly positive gain.
As it can be seen on (17), the expression of $\dot{C}_{e}$ is never singular: when moving in straight line on an even ground, equation (17) just leads to $\dot{C}_{e}=0$. As a result the cornering stiffness adaptation is just frozen and starts again, as soon as the robot turns. Adaptation law (17), contrarily to the one proposed in [12], does not require to monitor some singularity during straight line motion.

\section{LLT prediction}

The previous observer on-line supplies a realist estimation of $C_{e}$ and $\beta$ describing the grip conditions. All variables in model equations (3) and (5) are therefore available, and the LLT can then be predicted by integrating these equations over some temporal horizon $H$. If this prediction reaches a value superior than the threshold (i.e $L L T_{\text {predicted }}>0.8$ ), the driver is warned of a rollover risk.

More precisely, to perform the integration, the slowvarying variables, i.e the cornering stiffness $C_{e}$ and the bank angle $\theta$, are supposed constant over the horizon $H$. On the contrary, the driver's inputs (i.e the steering angle $\delta$ and the speed $v$ ) have an important influence on the short term evolution of the LLT. Therefore, it is proposed to extrapolate them using a linear function if $v$ and/or $\delta$ present an evolution rising the instability. Otherwise they are kept constant over the horizon $H$. In this way, the values of the LLT predicted from equations (3) and (5) are at worst overestimated, as it suits for a security device. Precisely, the extrapolation law that has been chosen is:

$$
\begin{aligned}
& v\left(t_{p}\right)=\left\{\begin{array}{l}
v(t)+d t \cdot \dot{v}(t), \text { if }(v(t), \dot{v}(t))>(0,0) \\
v(t) \text { otherwise }
\end{array}\right. \\
& \delta\left(t_{p}\right)=\left\{\begin{array}{l}
\delta(t)+d t \cdot \dot{\delta}(t), \text { if }(\delta(t), \dot{\delta}(t))>(0,0) \\
\delta(t)+d t \cdot \dot{\delta}(t), \text { if }(\delta(t), \dot{\delta}(t))<(0,0) \\
\delta(t) \text { otherwise }
\end{array}\right.
\end{aligned}
$$

with $t_{p}=t+d t$ and $0<d t<H$

\section{RESUlts}

\section{A. Setup testbed}

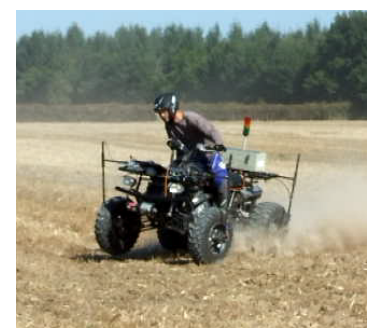

Fig. 4. MF400H, Massey Fergusson quad bike used for experiments.

In order to validate the observer and the relevance of the LLT prediction proposed in section III, experimental results are presented. They have been performed with a quad bike MF400H, manufactured by Massey Fergusson and depicted on Fig.4. Its dynamic parameters $m, I_{z}, k_{r}, b_{r}, h, a$ and $b$ have been preliminary calibrated, and it is equipped with the following sensors: 
- a Xsens MTI IMU providing accelerations and angular velocities.

- a Doppler radar supplying the linear speed

- an angular sensor providing the steering angle

This set of sensors constitutes a low cost perception system (compared to the ATV cost) enabling LLT estimation without requiring for expensive sensors. In addition, dynamometric sensors supplying tire/ground forces have been set up at each wheel. They provide a ground truth, but are not used in the algorithm.

\section{B. Experimental results}

1) ATV experimental path: The path described on Fig.5 has been performed on a mixed flat and sloping wet grass ground, at a speed between $3 \mathrm{~m} \mathrm{~s}^{-1}$ and $5 \mathrm{~m} \mathrm{~s}^{-1}$. It is composed of a straight line part executed on a $-15^{\circ}$ sloping ground, a U-turn on an even area and a second straight line on the same sloping ground.

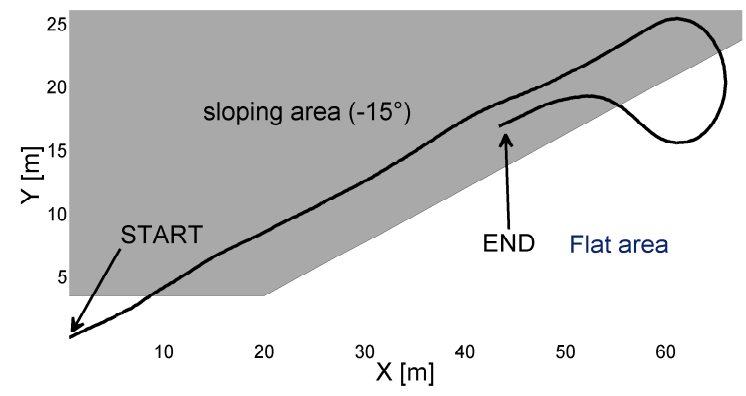

Fig. 5. ATV experiment path.

2) Estimated bank angle: On Fig.6, the bank angle profile estimated during experiments is depicted. It corresponds to actual slope values recorded $( \pm 15)$ previously by an operator. It then constitutes a sufficient estimation. The estimation inaccuracies in bank angle are indeed not critical since they will be compensated by the cornering stiffness adaptation.

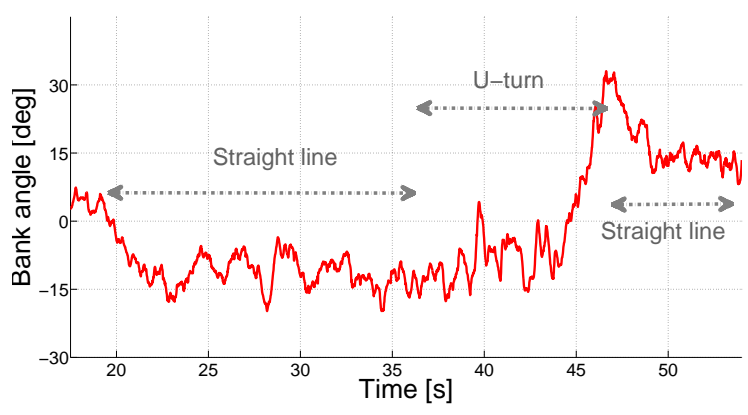

Fig. 6. Estimated bank angle.

3) Observer dynamics: Three experiments have been achieved with different initial conditions for the tire cornering stiffness: $60000 \mathrm{~N} \mathrm{rad}^{-1}, 20000 \mathrm{~N} \mathrm{rad}^{-1}, 2000 \mathrm{~N} \mathrm{rad}^{-1}$. The estimated tire cornering stiffnesses are then represented on Fig.7.

First, the estimated cornering stiffnesses converge all to the same value, this demonstrates that the choice of the initial

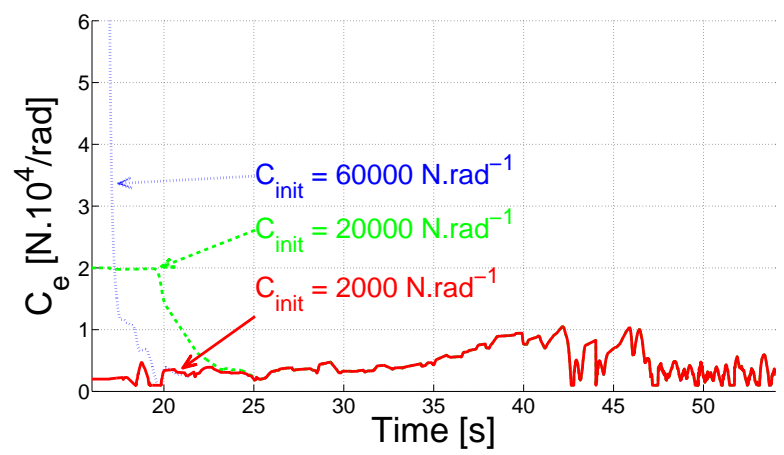

Fig. 7. Tire stiffness adaptation.

condition, which is uneasy, is satisfactorily not crucial. Moreover, the order of magnitude $\left(4000 \mathrm{~N} \mathrm{rad}^{-1}\right)$ is representative of the value for wet grass terrain considering a quad bike.

Secondly, the cornering stiffnesses are convergent despite of the straight line ( $17 \mathrm{~s}$ to $36 \mathrm{~s}$ ) due to the small slope. It generates sufficiently sideslip (see (14)) to adapt the cornering stiffness.

Finally $C_{e}$ suddenly decreases at $42 \mathrm{~s}$ (corresponding to an inversion of the steering angle sign), which is representative of non linear tire behavior when slip angle changes quickly.

4) Slope influence on the LLT estimation: On Fig.8 the LLTs estimated at the current instant (i.e. when $\mathrm{H}=0 \mathrm{~s}$ ) with and without the bank angle are depicted (respectively in solid and dashed line) and they are compared to the LLT measured thanks to dynamometric sensors. The quad bike enters the slope at $17 \mathrm{~s}$, then the U-turn occurs between $36 \mathrm{~s}$ and $46 \mathrm{~s}$, and finally the vehicle comes back on the slope part.

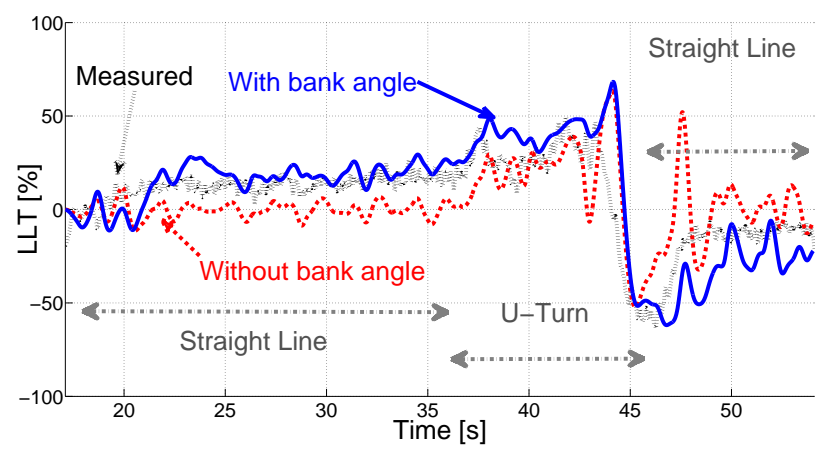

Fig. 8. Experiment results of the LLT estimation.

First, the LLT estimated without accounting for the bank angle stays around $0^{\circ}$ in straight line parts, as expected. In this case, $\theta$ is indeed mainly responsible of the LLT. Secondly, an overshoot can be observed from $48 \mathrm{~s}$ to $49 \mathrm{~s}$ due to the neglected slope too. On the contrary, the LLT accounting for the bank angle is almost superposed on the actual LLT, especially during the passage on the slope ( $17 \mathrm{~s}$ to $36 \mathrm{~s}$ ). And both the estimated LLT (with and without bank angle) are superposed as soon as ATV is on flat ground.

Nevertheless it can be observed some local inaccuracies, which are mainly due to the driver's behavior, neglected in 
the approach. Since quad bikes are light vehicles, the driver mass is important (for this experiment the driver's mass represents $25 \%$ of the total mass), and his behavior has a significant influence. As demonstrated in [17], the position of the driver may change significantly the location of the center of gravity of the overall system and consequently impacts the LLT values. This explains the slight errors in the LLT estimation in the turn-about ( $44 \mathrm{~s}$ to $46 \mathrm{~s}$ ) and during the movement back toward the start point ( $46 \mathrm{~s}$ to $54 \mathrm{~s}$ ).

This experiment shows the importance of taking into account the slope to estimate accurately the LLT. But the driver has to be informed of the risk before it appears, therefore a prediction algorithm is mandatory. The next section discusses the efficiency of the prediction algorithm developed in III-C.

5) Rollover risk indicator: The LLT estimated with the bank angle and the measured LLT are plotted again on Fig.9, respectively in blue and black lines, and compared to the LLT predicted with $H=1 s$ (shown in red line) .

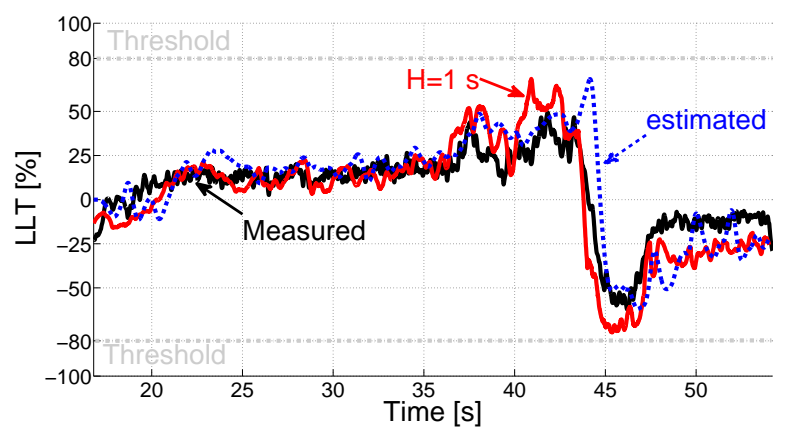

Fig. 9. Experiment results of the LLT prediction.

First, it can noticed that the three curves are almost superposed in steady state conditions: during the initial straight line part (up to $36 \mathrm{~s}$ ) and during the constant curve from $37 \mathrm{~s}$ to $40 \mathrm{~s}$. As expected since the ATV motion is then stationary: the predicted LLT is identical to the current value.

In contrast, during the transient phase ( $40 \mathrm{~s}$ to $46 \mathrm{~s}$ ) where rollover may occur, the predicted LLT satisfactorily precedes and overestimates the actual LLT.

Consequently, this rollover indicator is able to prevent the lift-off risk for the ATVs on natural ground.

\section{CONCLUSION AND FUTURE WORK}

This paper proposes an algorithm able to estimate and predict a rollover risk indicator for ATVs motion on natural ground. An adapted backstepping observer, based on a bicycle model, has been designed in order to estimate the dynamic variables (sideslip angle, cornering stiffness) allowing to adapt to varying conditions. Then, relying on a roll model, the LLT is on-line anticipated. The main contributions lie in the consideration of the terrain slope and in the grip condition adaptation. As demonstrated in the experiments, the LLT can be predicted accurately whatever the terrain conditions are (sliding, slope). Moreover the sensing equipment is limited to low cost sensors excluding expensive INS or
GPS. Nevertheless the driver's behavior, which influences the estimated LLT, is not taken into account. Therefore, in order to avoid unnecessary warnings, current developments aim at incorporating unused accelerometer/gyrometer data to account also for the driver's behavior.

\section{REFERENCES}

[1] "All terrain vehicle enforcement and safety report 2006," Wisconsin department of natural resources, USA, Tech. Rep., 2007.

[2] CCMSA, "Accidents du travail des salariés et non salariés agricoles avec des quads," Observatoire des risques professionels et du machinisme agricole, Paris, France, Tech. Rep., 2006.

[3] R. Bosch, Safety, comfort and convenience systems. Hoboken, U.S.A.: Wiley, 2006.

[4] J. Ackermann and D. Odenthal, "Advantages of active steering for vehicle dynamics control," in International Conference on Advances in Vehicle Control and Safety, Amiens: France, 1998.

[5] D. Bevly, J. Ryu, and J. Gerdes, "Integrating INS sensors with GPS measurements for continuous estimation of vehicle sideslip, roll, and tire cornering stiffness," IEEE Trans. Intell. Transport. Syst., vol. 7, no. 4, pp. $483-493,2006$.

[6] D. Piyabongkarn, R. Rajamani, J. Grogg, and J. Lew, "Development and experimental evaluation of a slip angle estimator for vehicle stability control," IEEE Trans. Contr. Syst. Technol., vol. 17, no. 1, pp. $78-88,2009$.

[7] B. Schofield, T. Hagglund, and A. Rantzer, "Vehicle dynamics control and controller allocation for rollover prevention," in IEEE International Conference on Control Applications, 2006, pp. 149 -154.

[8] G. Besseron, C. Grand, F. Ben Amar, and P. Bidaud, "Decoupled control of the high mobility robot hylos based on a dynamic stability margin," in IEEE/RSJ International Conference on Intelligent Robots and Systems (IROS), 2008, pp. $2435-2440$.

[9] K. Ohno, V. Chun, T. Yuzawa, E. Takeuchi, S. Tadokoro, T. Yoshida, and E. Koyanagi, "Rollover avoidance using a stability margin for a tracked vehicle with sub-tracks," in IEEE/RSJ Safety International Workshop on Security Rescue Robotics, 2009, pp. 1 -6.

[10] S. Peters and K. Iagnemma, "An analysis of rollover stability measurement for high-speed mobile robots," in Robotics and Automation, ICRA, May 2006, pp. $3711-3716$.

[11] M. Spenko, Y. Kuroda, S. Dubowsky, and K. Iagnemma, "Hazard avoidance for high-speed mobile robots in rough terrain," Journal of Field Robotics, vol. 23, no. 5, pp. 311-331, 2006.

[12] N. Bouton, R. Lenain, B. Thuilot, and P. Martinet, "A rollover indicator based on a tire stiffness backstepping observer: Application to an all-terrain vehicle," in Int. Conf. on Intelligent RObots and Systems (IROS), Nice, France, 2008.

[13] S. Peters, "Stability measurement of high-speed vehicles," Vehicle System Dynamics, vol. 47, no. 6, pp. 701-720, 2009.

[14] H. B. Pacejka, Tire and vehicle dynamics. Society of Automotive Engineers, 2002.

[15] C. Canudas de Wit and P. Tsiotras, "Dynamic tire friction models for vehicle traction control," in Decision and Control, vol. 4, 1999, pp. $3746-3751$ vol. 4 .

[16] K. Astrom and B. Wittenmark, Adaptive control (2nd edition). New York: Addison-Wesley, 1994.

[17] N. Bouton, "All-terrain vehicles dynamic stability. new solutions: Application to light vehicles like quad bike," Ph.D. dissertation, Blaise Pascal University, 2009. 